\title{
Zur Frage nach der physikalischen Bedeutung der Relativitätstheorie.
}

Von N. r. Raschersky in Prag.

(Eingegangen am 9. Juni 1922.)

In einer Reihe in letzter Zeit erschienener Abhandlungen hat Herr Guillaume1) den interessanten Versuch gemacht, der LorentzTransformation samt allen anderen daraus sich ergebenden Schlüssen der Relativitätstheorie im Sinne der klassischen Vorstellungen über Raum und Zeit eine physikalische Interpretierung zu geben. In seinen Betrachtungen behält Herr Gaillaume das Prinzip der Konstanz der Lichtgeschwindigkeit und ihrer Unabhängigkeit von der Geschwindigkeit der lichtemittierenden Quelle bei. Neben dieser konstanten Lichtgeschwindigkeit führt Herr Guilla ume noch eine „momentane Übergangsgeschwindigkeit"2) im Augenblick der Lichtemission ein, deren physikalische Auffassang unseres Wissens einige Schwierigkeiten und Bedenkungen bietet. Unter diesen Voraussetzungen kommt Herr Guillaume zum Schluß, daß die Relativitätstheorie nicht wahre Bewegungen, sondern scheinbare Bewegungen mit Aberrationen ${ }^{3}$ ) darstellt. Der Zusammenhang der scheinbaren Geschwindickeiten mit den wuhren ist dabei nicht näher festgestellt.

Das Hodograph der momentanen Lichtgeschwindigkeit ist ein Rotationsellipsoid mit der großen Achse parallel zur Translationsrichtung und der Leuchtquelle im hinteren Brenupunkte. Die Cbergangsgeschwindigkeit selbst bat bei Herrn Guillal ume eine rein formelle, mehr geometrische Bedentung and maß sogar in gewissem Sinne als eine rein mathematische Fiktion t) angesehen werden. Zweck der vorliegenden Untersuchung ist, eine phssikalisehe Interpretiorung der Relativitätstheorie zu geben, wobei von der Konstanz der Lichtgeschwindigkeit Abstand genommen wird. Auf der von dem Verfasser $\left.{ }^{5}\right)$ veröffentlichten Hypothese des verstorbenen Paschsky fußend, bat er versucht, dio Elektrodynamik bewegter Systeme zu

1) Guillaume n. Willigeus, Phys. Zs. 22, 909, 1921. Dort ist auch die weitere Literatur angegeben.

2) Phys. ZS. 22, 386, 1921. Guillaume, La théorie de la Relativité, Lausanne, F. Rouge \& Co., Editeurs, 1921, S. 15-16.

3) Phys. ZS. 22, 113, 1921.

4) Ebenda, S. 387 .

5) N. Raschevsky, Lightemission from a moving source. Phys. Rev. 18, 369 , November 1921.

Zeitgchrift für Physik. Bd. X. 
begründen 1). Das Paschsky-Prinzip lautet, daß das Hodograph der Lichtgeschwindigkeit, auf mit der Lichtquelle fest verbundene Achsen bezogen, ein Rotationsellipsoid mit der großen Achse parallel der Translationsrichtung, dem Leuchtpunkte in einem der Brennpunkte und der Exzentrizität gleich einer unbestimmten Funktion der absoluten Geschwindigkeit ist. Dabei ist aber von der reellen Lichtgeschwindigkeit in gewöhnlichem Sinne des Wortes die Rede, so daß diese Lichtgeschwindigkeit auf andere Achsen bezogen eine Funktion der absoluten Geschwindigkeit der Quelle sowohl wie dieser Achsen ist.

Wir beabsichtigen nun zu zeigen, daß unter Zugrundelegung dieser Anschauung und Berücksichtigung der Tatsache, daß wir die wahre, "unidirektionale" (s. weiter unten) Lichtgeschwindigkeit nicht messen können, der Úbergang von einem bewegten System zum anderen sich durch eine der Lorentzsehen analoge Transformation darstellt.

1. Begründung des Paschsky-Prinzips. Wir geben in diesem Paragraphen kurz die Begrïndung des Prinzips, ungefähr in der Weise, wie sie in den Manuskripten des Verstorbenen enthalten ist ${ }^{2}$ ).

Im Gegensatz zu der Voraussetzung der Konstanz der Lichtgeschwindigkeit legen wir nnseren Betrachtungen das folgende Postulat zugrunde: die Geschwindigkeit des von einer bewegten Quelle ausgesandten Lichtes sei, bezogen auf absolut ruhende Achsen, gleich

$$
c+f_{1}(v)
$$

in der Richtang der Geschwindigkeit und

$$
c+f_{2}(v)
$$

in der entgegengesetzten Richtung, wobei

$$
f_{1}(v) \text { und } f_{2}(v)
$$

zwei beliebige Funktionen, endliche und stetige, der absoluten Translationsgeschwindigkeit $v$ sind, mit der einzigen Bedingung, daß

$$
f_{1}(0)=f_{2}(0)=0
$$

$c$ bedeutet dabei die Lichtgeschwindigkeit von einer ruhenden Quelle, auf rahende Achsen bezogen.

In bezug auf ein mit den Achsen bewegtes Koordinatensystem werden diese Geschwindigkeiten gleich

$$
\left.\begin{array}{rl}
c+v+f_{2}(v) & =\mathfrak{l}_{2}(v)=\sigma+F_{2}(v) \\
c-v+f_{1}(v) & =\mathfrak{l}_{1}(v)=\sigma+F_{1}(v)
\end{array}\right\}
$$

In diesen Ausdrücken bedentet $\sigma$ eine Funktion von $v$ mit der Eigenschaft, daß

1) Phys. Z8. 23, 2, 1922.

2) Vgl. Phys. Rev., 1. c. 
Zur Frage nach der physikalischen Bedeutung der Relativitätstheorie. 211

ist und $F_{1}(v)$ und $F_{2}(v)$ zwei andere beliebige stetige Funktionen von $v$ mit der Eigenschaft

$$
F_{1}(0)=F_{2}(0)=0 .
$$

Hier wird es von Nutzen sein, genauer zu definieren, was wir unter dem Wort „Lichtgeschwindigkeit" zu verstehen baben. Alle bis jetzt benutzten Methoden, um diese Geschwindigkeit zu ermitteln, beruhen anf den Prinzipien des Fizeauschen oder Foucaultschen Versuchs. In beiden durchläuft das Licht einen gegebenen Weg zweimal in entgegengesetzten Richtungen.

Die Zeit, welche das Licht braucht, un von einem Punkte ausgehend nach erfolgter Reflexion wieder nach dem Anfangspunkt zurückzukehren, wird durch die Formel

$$
T=\frac{2 l}{\sigma}
$$

gegeben, worin $l$ die Länge des Weges bedeutet.

$\sigma$ ist jedoch keineswegs die wahre Lichtgeschwindigkeit, da die Geschwindigkeiten in beiden Richtungen verschieden sein können. Die so gefundene Geschwindigkeit nennen wir die ,berechnete Lichtgeschwindigkeit" zum Unterschied von der "wahren Lichtgeschwindigkeit", die nur durch eine Methode ermittelt werden könnte, welche die Lichtfortpflanzung in einer Richtung benutzt.

Unsere bisherigen physikalischen Methoden liefern nur die „berechnete Lichtgeschwindigkeit". Das ist sehr wichtig.

Da aber unsere Hypothese den Ergebnissen des Michelson-Versuchs Rechnung tragen muß, welches eigentlich nichts mehr aussagt, als daß die "berechnete Lichtgeschwindigkeit" von der Richtung unabhängig ist, müssen wir fordern, daß die Zeit, welche das Licht bedarf, um von einem Pankt $A$ zum anderen Punkt $B$ zu gelangen, unabhängig davon ist, ob das System sich in der Richtung $A B$ oler $B A$ bewegt (wenn $A B$ parallel $v$ ist) und den Wert

$$
T=\frac{2 l}{\sigma}
$$

bat, also haben wir

$$
\frac{l}{\sigma+F_{1}(v)}+\frac{l}{\sigma+} \overline{F_{2}(v)}=\frac{2 l}{\sigma}
$$

oder nach Umrechnung

Setzen wir

$$
\begin{gathered}
-\sigma / 2\left[F_{1}(v)+F_{2}(v)\right]=F_{1}(v) F_{2}(v) . \\
F_{1}(v)+F_{2}(v)=2 \psi(v),
\end{gathered}
$$

wo $\psi(v)$ eine beliebige endliche stetige Fanktion von $v$ sein maß mit der Bedingung

$$
\psi(0)=0,
$$


so erhalten wir

$$
\left.\begin{array}{r}
-\sigma \psi(v)=F_{1}(v) \cdot F_{2}(v) \\
2 \psi(v)=F_{1}(v)+F_{2}(v)
\end{array}\right\}
$$

Daraus folgt, daß $F_{1}(v)$ und $F_{2}(v)$ die Wurzeln der quadratischen Gleichnng

sind. Also

$$
-F^{2}+2 \psi(v) F+\sigma \psi(v)=0
$$

$$
\left.\begin{array}{l}
F_{1}(v)=\psi(v)+\sqrt{\psi^{2}(v)+\sigma \psi(v)} \\
F_{2}(v)=\psi(v)-\sqrt{\psi^{2}(v)+\sigma \psi(v)}
\end{array}\right\}
$$

und für Lichtgeschwindigkeiten in bezug auf mitbewegte Achsen bekommen wir

$$
\left.\begin{array}{l}
\sigma+\psi+\sqrt{\psi^{2}+\sigma \psi} \\
\sigma+\psi-\sqrt{\psi^{2}+\sigma \psi}
\end{array}\right\}
$$

[Hier ist anstatt $\psi(v)$ wie auch in weiterem $\psi$ gebraucht.]

Wir sehen aber aus (3), daß wir die Rollen der Funktionen $F_{1}(v)$ and $F_{2}(v)$ vertauschen können. Also bekommen wir

$$
\left.\begin{array}{l}
\sigma+\psi+\sqrt{\psi^{2}+\sigma \psi} \\
\sigma+\psi+\sqrt{\psi^{2}+\sigma \psi}
\end{array}\right\}
$$

Bisher ließen wir die Lichtgeschwindigkeit in den Richtangen, welche einen beliebigen Winkel mit der Translationsrichtung bilden, unberücksichtigt. Wir können aber zeigen, daß man mit dem Michelsonschen Versuch nicht in Widerspruch kommt, falls man folgende Hypothese macht:

Das Hodograph der Lichtgeschwindigkeiten, bezogen auf ein mitbewegtes Achsensystem, sei ein Rotationsellipsoid mit der großen Achse als Rotationsachse, welche auch parallel der Translationsrichtung liegt. Die Quelle liege in einem der Brennpunkte des Ellipsoids, die Lichtgeschwindigkeiten parallel der Translationsgeschwindigkeit seien durch (9) gegeben.

Dann haben wir für die große Halbachse

$$
a=\sigma+\psi \text {. }
$$

Die lineare Exzentrizität ist gleich

$$
e=\sqrt{\psi^{2}+6 \psi} \text {. }
$$

Der Parameter, welcher die Lichtgeschwindigkeit in der zur Translationsrichtung senkrechten Richtung angibt, ist gleich

$$
p=\sigma
$$

und die numerische Exzentrizität wird

$$
\frac{e}{a}=\sqrt{\frac{\psi}{\sigma+\psi}}=k
$$


Aus diesen Formeln folgt, daß die Lichtgeschwindigkeit in einer Richtung, welche mit der Translationsrichtung den Winkel $\varphi$ einschließt, durch

gegeben ist.

$$
\sigma_{\varphi}=\frac{\sigma}{1 \pm \sqrt{\frac{\psi}{\sigma+\psi} \cos \varphi}}
$$

Das positive Zeichen im Nenner entspricht der Annahme, daß die Quelle im Vorderbrennpunkte, das negative, daß sie im Hinterbrennpunkte liegt. Falls wir annehmen, daß jeder reflektierende Pankt sich wie eine unabhängige Quelle verhält (Huygenssches Prinzip), so können wir leicht einsehen, daß unsere Hypothese den Michelsonschen Versuch erklärt.

Die Zeit, welche das Licht braucht, um vom Zentrum bis za einem gegebenen Punkte $A$ einer mit der Lichtquelle mitbewegten Kugel zu kommen, ist in der Tat gleich

$$
T_{1}=\frac{l}{\sigma_{\varphi}}=\frac{l(1 \pm k \cos \varphi)}{\sigma},
$$

wobei $\varphi$ der Winkel ist, welchen der vom Mittelpunkte gezogene Radius mit $v$ bildet. Der zweite Teil des Weges wird in der Zeit

$$
T_{2}=\frac{l}{\sigma_{p+\pi}}=\frac{l(1 \mp k \cos \varphi)}{\sigma}
$$

durchlaufen. Die Vorzeichen bei $k$ sind vertauscht, weil

$$
\cos (\varphi+\pi)=-\cos \varphi
$$

ist. Die Gesamtzeit, welche der Lichtstrahl braucht, um hin und her zu laufen, ist gleich

$$
T=T_{1}+T_{2}=\frac{2 l}{\sigma}
$$

und unabhängig von der Richtung des Strahles.

Formel (12) zeigt auch, daß, falls wir die Lichtgeschwindigkeit mit den üblichen Methoden messen, sie immer gleich $\sigma$ gefunden wird.

$\sigma$ ist die "berechnete" Lichtgesehwindigkeit im System und ist innerhalb des gegebenen Systems eine Konstante. Ist allgemein

$$
\sigma=\text { Const }=c \text {, }
$$

so sind wir nicht imstande, durch Ermittlung des absoluten Betrages der "berechneten" Lichtgeschwindigkeit die absolute Bewegung des Systems festzustellen. Anderenfalls ist es prinzipiell möglich, aber auch nur unter der Voraussetzang, daß die Geschwindigkeit des Systems zeitlich nicht immer denselben Wert behält, also zuerst einen Wert $v_{1}$ hatte und dann einen anderen Wert $v_{2}$ annimmt. 
$\mathrm{Da}$

$$
\psi \text { und } k=\sqrt{\frac{\psi}{\sigma+\psi}}
$$

beliebige, endliche, stetige Funktionen sind, die nur der Bedingung

$$
\psi(0)=k(0)=0
$$

unterworfen sind, so haben wir eine unendliche Zabl von Lösungen des Michelsonschen Problems gefunden.

2. Stellen wir uns jetzt ein gleichförmig geradlinig bewegtes System vor. Die sich in diesem System befindenden Beobachter können, wie wir gesehen haben, nur die „berechnete Lichtgeschwindigkeit" б ermitteln. Die wirklich vorhandene optische Anisotropie des Raumes bleibt ihnen. verborgen. Da nichts zu einer entgegengesetzten Annahme zwingt, werden unsere Beobachter natürlich glauben, daß die "wahre" Lichtgeschwindigkeit auch nach allen Richtungen denselben Wert $\sigma$ hat, und daß der Raum optisch nicht nur scheinbar, sondern wirklich ganz isotrop ist.

Diese Annahme ist aber einer anderen äquivalent, nämlich derjenigen, daß eine Uhr, deren Abszisse relativ zum Leuchtpunkte gleich $x$ ist, nicht die wahre universelle Zeit $t$ anzeigt, sondern eine andere lokale Zeit $\tau^{\prime}$, die mit $t$ durch die Gleichung verknüpft ist

$$
\tau^{\prime}=t \mp \frac{k}{\sigma} x \text {. }
$$

In der Tat betrachten wir eine Kugel vom Radius $l$ in unserem System, deren Mittelpunkt mit dem Koordinatennullpunkt zusammenfällt und in dem sich der Leuchtpunkt befindet. Im Zeitmoment 0 emittiere unser Leuchtpankt ein Lichtsignal. Die Ankunftszeit des Signals auf der Kugel ist durch

$$
\frac{l(1+k \cos \varphi)}{\sigma}
$$

gegeben; führen wir jetzt die lokale Zeit $\tau^{\prime}$ ein, so wird das betrachtete Zeitintervall gleich

$$
\frac{l(1+k \cos \varphi)}{\sigma}+\frac{k}{\sigma} l \cos \varphi=\frac{l}{\sigma} .
$$

Bei Benutzung der Lokalzeit $\tau^{\prime}$ ist also wirklich die "wabre Geschwindigkeit" nach allen Richtungen gleich $\sigma$.

Die Beobachter in unserem System gebrauchen somit bei allen ihren physikalischen Messungen und bei der Beschreibung aller physikalischen Erscheinungen nicht die universelle, sondern die lokale Zeit.

Daher werden allgemein alle beobachteten Geschwindigkeiten nur scheinbar sein, denn sie werden durch die Derivierte $d x d \tau^{\prime}$ 
Zur Frage nach der physikalischen Bedeutung der Relativitätstheorie. 215

gegeben, während die wahre Geschwindigkeit durch $d x ; d t$ ansgedrückt ist.

Untersuchen wir nun, wie sich diese scheinbaren Geschwindigkeiten durch die wahren ausdrücken.

Betrachten wir dazu zwei Systeme $S_{1}$ und $S_{2}$. Wir wählen dabei das Achsenkreuz so, daß die $x$-Achsen parallel der relativen Geschwindigkeit von $S_{1}$ und $S_{2}$ sind. Zwecks Vereinfachung betrachten wir zuerst den Fall, daß $v_{1}\left\|v_{2}\right\| v_{12}$ ist. Später werden wir diese Beschränkung fallen lassen. Seien die Koordinaten, Lokalzeiten, absolute Geschwindigkeiten und die betreffenden Funktionen $k$ und $\sigma$ bzw. gleich

$$
x_{1}, y_{1}, z_{1}, \tau_{1}^{\prime}, v_{1}, k_{1}, \sigma_{1} \text { und } x_{2}, y_{2}, z_{2}, \tau_{2}^{\prime}, v_{2}, k_{2}, \sigma_{2},
$$

und bezeichnen wir ferner

$$
v_{2}-v_{1}=v_{12}=-v_{21} \text {. }
$$

Wir nehmen roch die Richtung von $v_{1}$ als positiv an und zählen jede Geschwindigkeit positiv oder negativ, je nachdem sie zu $v_{1}$ gleich oder entgegengerichtet ist.

$k$ muß dann auch positiv oder negativ sein, je nachdem $v$ negativ oder positiv ist. Ist also z. B. $v_{1}$ dem absoluten Betrage nach gleich $v_{2}$, aber entgegengerichtet, so müssen wir für das Hodographellipsoid baben

$$
\sigma_{1 \varphi}=\frac{\sigma}{1 \pm k_{1} \cos \varphi} ; \quad \sigma_{2 \varphi}=\frac{\sigma}{1 \pm k_{2} \cos \varphi}=\frac{\sigma}{1 \mp k_{1} \cos \varphi} .
$$

Wir nehmen $k$ immer vom gleichen Vorzeichen wie $v$ an. Dann haben wir die Relationen

$$
\left.\begin{array}{c}
v_{12}=\frac{d x_{1}}{d t} \\
\tau_{1}^{\prime}=t \mp \frac{k_{1}}{\sigma_{1}} x_{1} ; \quad \tau_{2}^{\prime}=t \mp \frac{k_{2}}{\sigma_{2}} x_{2} \\
t=\tau_{1}^{\prime} \pm \frac{k_{1}}{\sigma_{1}} x_{1}=\tau_{2}^{\prime} \pm \frac{k_{2}}{\sigma_{2}} x_{2}
\end{array}\right\}
$$

Die zugehörigen scheinbaren Geschwindigkeiten bezeichnen wir durch

Es ist also

$$
u_{12}^{\prime} ; \quad u_{21}^{\prime} \text {. }
$$

$$
u_{12}^{\prime}=\frac{d x_{1}}{d \tau_{1}^{\prime}} \text {. }
$$

Nun haben wir aber

oder

$$
\frac{d x_{1}}{d \tau_{1}^{\prime}}=\frac{d x_{1}}{d t} \cdot \frac{d t}{d \tau_{1}^{\prime}}=\frac{d x_{1}}{d t}\left(1 \pm \frac{k_{1}}{\sigma_{1}} \frac{d x_{1}}{d \tau_{1}^{\prime}}\right)
$$

$$
u_{12}^{\prime}=v_{12}\left(1 \pm \frac{k_{1}}{\sigma_{1}} u_{12}^{\prime}\right) \text {. }
$$


Daraus erhalten wir leicht

Analog

$$
u_{12}^{\prime}=\frac{v_{12}}{1 \mp \frac{k_{1}}{\sigma_{1}} v_{12}} .
$$

Wir sehen, daß allgemein $u_{12}^{\prime} \lessgtr u_{21}^{\prime}$.

$$
u_{21}^{\prime}=\frac{v_{21}}{1 \mp \frac{k_{2}}{\sigma_{2}} v_{21}}=\frac{-v_{12}}{1 \pm \frac{k_{2}}{\sigma_{2}} v_{12}} \text {. }
$$

$\mathrm{Um}$ die Symmetrieeigenschaft $\mathrm{zn}$ erhalten, $\mathrm{nm}$ also $u_{12}^{\prime}=-u_{21}^{\prime}$ schreiben za können, müssen wir

$$
1 \mp \frac{k_{1}}{\sigma_{1}} v_{12}=1 \pm \frac{k_{2}}{\sigma_{2}} v_{12} \text { oder } \frac{k_{1}}{k_{2}}=-\frac{\sigma_{1}}{\sigma_{2}}
$$

setzen, was physikalisch sinnlos ist.

Wir werden nun aber sehen, daß die Symmetrie erhalten werden kann bei einer etwas verschiedeneren Betrachtung des Problems der Zeitbestimmung.

Das $\sigma$ kann nur dann als Funktion von $v$ angeseben werden, wenn wir uns als "Zentraluhr" eines Vorganges bedienen, der unabhängig von der Lichtfortpflanzungsgeschwindigkeit ist. So ist z. B. die Erde die Zentralubr der mechanischen Vorgänge ${ }^{1}$ ). Nelimen wir aber, analog den Betrachtungen Guillaumes, das Licht ais Zentraluhr aller physikalischen Vorgänge an, so definieren wir nnsere Zeiteinheit dadurch, daß wir für die Lichtgeschwindigkeit einen gewissen konstanten $W$ ert $\sigma_{0}$ setzen. In der Wahl dieses Wertes $\sigma_{0}$ sind wir natürlich ganz frei und wir setzen

$$
\sigma_{0}=3 \cdot 10^{10} \mathrm{~cm} / \mathrm{sec}=c \text {. }
$$

Wir betonen aber bier, daß die oben erwähnte Behauptung nar eine willkürliche ist, und dieser willkürliche, sozusagen konventionelle Charakter haftet daher auch den folgenden Schlüssen an.

Dies vorausgesetzt, sehen wir, daß unsere Beobachter im bewegten System für die wabre Lichtgeschwindigkeit den Wert $\sigma_{0}$ annehmen werden.

Daher wird auch die von diesen Beobachtern festgesetzte Zeiteinheit von der oben betrachteten Lokalzeiteinbeit verschieden sein. Der Zusammenhang dieser neuen Zeit $\tau$ mit der Lokalzeit $\tau^{\prime}$ ist aber leicht zu ermitteln. Wir baben nämlich nach Definition

wo $l$ der Lichtweg ist. Daraus

$$
\tau_{1}^{\prime}=\frac{l}{\sigma_{1}} ; \quad \tau_{1}=\frac{l}{\sigma_{0}},
$$

$$
\tau_{1}=\tau_{1}^{\prime} \frac{\sigma_{1}}{\sigma_{0}}
$$

1) Vgl. Guillanme, Phys. ZS. 22, 112, 1921. 
Zur Frage nach der physikalischen Bedeutung der Relativitätstheorie. 217

Jetzt sind also die Raum- und Zeitkoordinaten in unseren Systemen $S_{1}$ und $S_{2}$ bzw.

und

$$
x_{1} y_{1} z_{1} \tau_{1}=\frac{\sigma_{1}}{\sigma_{0}} \tau_{1}^{\prime}=\frac{\sigma_{1}}{\sigma_{0}} t \mp \frac{k_{1}}{\sigma_{0}} x_{1}
$$

$$
x_{2} y_{2} z_{2} \tau_{2}=\frac{\sigma_{2}}{\sigma_{0}} t \quad \mp \frac{k_{2}}{\sigma_{0}} x_{2}
$$

Also

$$
t=\frac{\sigma_{0}}{\sigma_{1}} \tau_{1} \pm \frac{k_{1}}{\sigma_{1}} x_{1}=\frac{\sigma_{0}}{\sigma_{2}} \tau_{2} \pm \frac{k_{2}}{\sigma_{2}} x_{2} .
$$

Wie oben, definieren wir anch hier die scheinbaren Geschwindigkeiten durch

und erhalten daher

$$
u=\frac{d x}{d \tau}
$$

oder

$$
\frac{d x_{1}}{d \tau_{1}}=\frac{d x_{1}}{d t} \cdot \frac{d t}{d \tau_{1}}=\frac{d x_{1}}{d t}\left(\frac{\sigma_{0}}{\sigma_{1}} \pm \frac{k_{1}}{\sigma_{1}} \frac{d x_{1}}{d \tau_{1}}\right)
$$

Daraus

$$
u_{12}=v_{12}\left(\frac{\sigma_{0}}{\sigma_{1}} \pm \frac{k_{1}}{\sigma_{1}} u_{12}\right)
$$

$$
u_{12}=\frac{\sigma_{0} v_{12}}{\sigma_{1} \mp k_{1} v_{12}}
$$

Wir leiten noch folgende Formel ab, die uns später von Nutzen sein wird

$$
\frac{u_{12}}{v_{12}} \frac{\sigma_{1}}{\sigma_{0}}=1 \pm \frac{k_{1}}{\sigma_{0}} u_{12}=\frac{1}{1 \mp \frac{k_{1}}{\sigma_{1}} v_{12}} .
$$

Analog (24) haben wir

$$
u_{21}=\frac{\sigma_{0} v_{21}}{\sigma_{2} \mp k_{2} v_{21}}=\frac{-\sigma_{0} v_{12}}{\sigma_{2} \pm k_{2} v_{12}} \text {. }
$$

Damit $u_{12}=-u_{21}$ ist, müssen wir setzen $\sigma_{1} \mp k_{1} v_{12}=\sigma_{2} \pm k_{2} v_{12}$ oder nach Umrechnung

woraus sich ergibt

$$
\sigma_{1}-\sigma_{2}= \pm\left(k_{1}+k_{2}\right) v_{12},
$$

$$
\frac{\sigma_{1}}{\sigma_{2}}=1 \pm \frac{k_{1}+k_{2}}{\sigma_{2}} v_{12} \text {. }
$$

Nehmen wir an, $S_{1}$ sei in absoluter Ruhe. Dann baben wir und (28) ergibt

$$
\sigma_{1}=\sigma_{0} ; \quad k_{1}=0 ; \quad v_{12}=v_{2}
$$

$$
\frac{\sigma_{0}}{\sigma_{2}}=1 \pm \frac{k_{2}}{\sigma_{2}} v_{2}
$$


In derselben Weise erhalten wir, indem wir jetzt $S_{2}$ als ruhend annehmen,

$$
\sigma_{2}=\sigma_{0} \quad k_{2}=0 \quad r_{12}=-v_{1} \quad \frac{\sigma_{1}}{\sigma_{0}}=1+\frac{k_{1}}{\sigma_{0}} v_{1} .
$$

Suchen wir jetzt das Additionstheorem der scheinbaren Geschwindigkeiten.

Aus (24) und (26) haben wir

und aus (23)

$$
u_{12}=\frac{\sigma_{0} v_{12}}{\sigma_{1} \mp k_{1} v_{12}} ; \quad u_{23}=\frac{\sigma_{0} v_{23}}{\sigma_{2} \mp k_{2} v_{23}} ; \quad u_{13}=\frac{\sigma_{0} v_{13}}{\sigma_{1} \mp k_{1} v_{13}}
$$

$$
v_{12}=\frac{\sigma_{1} u_{12}}{\sigma_{0} \pm k_{1} u_{12}} ; \quad v_{23}=\frac{\sigma_{2} u_{23}}{\sigma_{0}+k_{2} u_{23}} .
$$

$\mathrm{Da}$ für die wahren Geschwindigkeiten offenbar $v_{13}=v_{12}+v_{23}$ gilt, so ist ferner

$$
u_{13}=\frac{\sigma_{0}\left(v_{12}+v_{23}\right)}{\sigma_{1}+k_{1}\left(v_{12}+v_{23}\right)} .
$$

Setzen wir für $v_{12}$ und $v_{23}$ ihre Werte aus (32) ein, so erbalten wir nach Umrechnung

$$
u_{13}=\frac{\left(u_{12}+\frac{\sigma_{2}}{\sigma_{1}} u_{23}\right) \pm\left(\frac{k_{2}}{\sigma_{0}}+\frac{\sigma_{2}}{\sigma_{1}} \frac{k_{1}}{\sigma_{0}}\right) u_{12} u_{23}}{1 \pm\left(\frac{k_{2}}{\sigma_{0}}-\frac{\sigma_{2} l_{1}}{\sigma_{1}} \frac{k_{0}}{\sigma_{0}}\right) u_{23}-\frac{k_{1}^{2} \sigma_{2}}{\sigma_{0}^{2}} \frac{\sigma_{1}}{\sigma_{1}} u_{12} u u_{23}} .
$$

Um von dieser Formel Gebrauch machen za können, führen wir die rereinfachenden Annahmen ein, daß für kleine $u$ das Verhältnis $\sigma_{1} \sigma_{2}$ annähernd gleich 1 gesetzt, und daß das letzte Glied im Nenner wegen des Faktors $1 / \sigma_{\tilde{u}}^{2}$ vernachlässigt werden kann; dann bekommt unsere Formel die Gestalt

$$
u_{1 ; 3}=\frac{u_{12}+u_{23} \pm \frac{k_{1}+k_{2}}{\sigma_{0}} u_{12} u_{23}}{1 \pm \frac{k_{2}-k_{1}}{\sigma_{0}} u_{23}} .
$$

Diese Näherungsformel wenden wir auf den Fizealu-Fresnelschen Versuch an. Es sei $u$ die Geschwindigkeit des Wassers, $n$ sein Brechungskoeffizient, $q$ die gesuchte resultierende Geschwindigkeit.

Wir setzen

$$
u_{13}=q ; \quad u_{12}=w ; \quad u_{23}=\frac{\sigma_{0}}{n} ;
$$

dann erhalten wir

$$
q=\frac{w+\frac{\sigma_{0}}{n} \pm \frac{k_{1}+k_{2}}{n} w}{1 \pm \frac{k_{2}-k_{1}}{n}}
$$


Zur Frage nach der physikalischen Bedeutung der Relativitätstheorie. 219

Entwickeln wir diesen Ausdruck in eine Potenzreihe, wobei wir die Größen zweiter Ordnung vernachlässigen, so bekommen wir

$$
q=w+\frac{\sigma_{0}}{n} \mp \frac{\sigma_{0}\left(k_{2}-k_{1}\right)}{n^{2}} \pm \frac{k_{1}+k_{2}}{n} w \mp \frac{k_{2}-k_{1}}{n} v \mp \frac{k_{1}^{2}-k_{2}^{2}}{n^{2}} w .
$$

Da die Funktion $k$ im Vergleich mit $u$ sehr klein ist, sehen wir leicht ein, daß die drei letzten Glieder vernachlässigt werden können. Ist dabei annähernd $k_{2}-k_{1}$ gleich $w / \sigma_{0}$, so erhalten wir

$$
q=\frac{\sigma_{0}}{n}+w\left(1 \mp \frac{1}{n^{2}}\right) \text {. }
$$

Für den Fall des Minuszeichens ergibt sich die bekannte Fresnelsche Formel. Das Experiment zwingt uns also das Minuszeichen anzunebmen, welches dem Pluszeichen in der Gleichung (11) entspricht. Wir können also behaupten, daß die Lichtquelle im vorderen Brennpunkte des Ellipsoidbodographs liegt. Die Funktion $k$ kann auch näherungsweise von der Größenordnung $v / \sigma_{0}$ angenommen werden; über ihre wahre Gestalt sind wir aber zurzeit noch im Dunkeln.

In den folgenden Betrachtungen beschränken wir uns, um uns nicht in den Allgemeinheiten zu verlieren, auf den Fall, daß die Symmetriebedingungen (28), (29) und (30) erfüllt sind, obwohl auch diese Einschränkung nicht zwingend ist.

Die wahre relative Bewegung der beiden Systeme $S_{1}$ und $S_{2}$ ist durch die Galileische Transformation dargestellt

$$
x_{2}=x_{1}-v_{12} t ; \quad y_{2}=y_{1} ; \quad z_{2}=z_{1} ; \quad t=t .
$$

Führen wir in die erste dieser Gleichungen für $t$ seinen Wert in $\tau_{1}$ aus (21) ein, wobei wir jetzt nur das erste Vorzeicheu gebrauchen, so erhalten wir nach Umrechnung

$$
x_{2}=\left(1-\frac{k_{1}}{\sigma_{1}} v_{12}\right) x_{1}-v_{12} \frac{\sigma_{0}}{\sigma_{1}} \tau_{1}
$$

oder mit Berücksichtigung von (24)

oder nach (25)

$$
\begin{gathered}
x_{2}=\left(1-\frac{k_{1}}{\sigma_{1}} v_{12}\right)\left(x_{1}-u_{12} \tau_{1}\right) \\
x_{2}=\frac{x_{1}-u_{12} \tau_{1}}{1-\frac{k_{1}}{\sigma_{0}} u_{12}} .
\end{gathered}
$$

Die Analogie mit der ersten Lorentzschen Transformationsgleichung ist auffallend.

Fassen wir jetzt die Gleichung (21) ins Auge, die wir hier noch einmal hinschreiben

$$
\frac{\sigma_{0}}{\sigma_{1}} \tau_{1}+\frac{k_{1}}{\sigma_{1}} x_{1}=\frac{\sigma_{0}}{\sigma_{2}} \tau_{2}+\frac{k_{2}}{\sigma_{2}} x_{2}
$$


setzen wir für $x_{2}$ in dieser Formel seinen Ausdruck (40), in den wir noch

$$
\frac{1}{1+\frac{k_{1}}{\sigma_{0}} u_{12}}=\gamma_{1}=\frac{1}{\vartheta_{1}}
$$

einführen, so bekommen wir

Setzen wir noch

$$
\tau_{2}=\frac{\tau_{1}+\frac{\sigma_{2} \vartheta_{1}^{2}-\sigma_{1}}{\sigma_{1} u_{12}} x_{1}}{1+\frac{k_{1}}{\sigma_{0}} u_{12}}
$$

$$
\frac{\sigma_{2} \vartheta^{2}-\sigma_{1}}{\sigma_{1} u_{12}}=\chi\left(u_{12}, v_{1}\right)
$$

so erhalten wir, falls wir die lokale Zeit und die scheinbare Geschwindigkeit benutzen, folgendes System von Transformationsgleichungen

$x_{2}=\gamma_{1}\left(x_{1}-u_{12} \tau_{1}\right) ; \quad y_{2}=y_{1} ; \quad z_{2}=z_{1} ; \quad \tau_{2}=\gamma_{1}\left(\tau_{1}+\chi x_{1}\right)$.

Wir sehen, daß wir hier, wie anch in der Relativitätstheorie, die Formel im Sinne einer Kontraktion im Verhältnis $1 / \gamma_{1}$ interpretieren können. Aus der Ableitung der Formel aber geht hervor, daß von einer physischen, reellen Kontraktion gar keine Rede sein kann. Sie kann nur als mathematische Fiktion angesehen werden.

Um von den erhaltenen Transformationsgleichungen Gebrauch zu machen, berücksichtigen wir folgendes. Die Experimente lehren uns, daß bei Benntzung der lokalen Zeit, die uns eigentlich nur zugänglich ist, alle physikalischen Erscheinungen sich so abspielen, als ob das System in Rube wäre, d. $b$. daß es innerbalb des Systems unmöglich ist, seine absolute Bewegung zu entdecken. Indem wir also in die Gleichung eines physikalischen Vorganges, wie sie für ein ruhendes System lautet, für die $x_{1}, y_{1}, z_{1}, \tau_{1}$ als gültig. anvehmen and dann diese Gleichung mit Hilfe unserer Transformationsgleichungen (43) transformieren, finden wir, wie sich dieser Vorgang, vom System $S_{2}$ aus gesehen, abspielt.

Bevor wir zu weiteren Betrachtungen übergehen, sei hier noch untersucht, wie sich die Verbältnisse verändern, falls die absoluten Geschwindigkeiten beider Systeme einen beliebigen Winkel miteinander bilden.

Wählen wir die Achsenkreuze in der Weise, daß die X-Achsen parallel zu der relativen Geschwindigkeit $v_{12}$ sind und bezeichnen 
Zur Frage nach der physikalischen Bedeutung der Relativitätstheorie. 221

wir mit $\delta_{1}, \delta_{2}$ bzw. die Winkel zwischen $v_{12}$ und $v_{1}$ bzw. $v_{2}$, so ist offenbar anstatt der Ausdrücke (20) für $\tau_{1}$ und $\tau_{2}$ folgendes zu setzen

$$
\left.\begin{array}{l}
\tau_{1}=\frac{\sigma_{1}}{\sigma_{0}} t-\frac{k_{1}}{\sigma_{0}}\left(x_{1} \cos \delta_{1}-\sqrt{y_{1}^{2}+z_{1}^{2}} \sin \delta_{1}\right) \\
\tau_{2}=\frac{\sigma_{2}}{\sigma_{0}} t-\frac{k_{2}}{\sigma_{0}}\left(x_{2} \cos \delta_{2}-\sqrt{y_{3}^{2}+z_{2}^{2}} \sin \delta_{2}\right)
\end{array}\right\}
$$

Führen wir

ein, so ist

$$
\left.\begin{array}{l}
k_{1} \cos \delta_{1}=x_{1} ; \quad \frac{k_{1}}{\sigma_{0}} \sqrt{y_{1}^{2}+z_{1}^{2}} \sin \delta_{1}=A \\
k_{2} \cos \delta_{2}=x_{2} ; \quad \frac{k_{2}}{\sigma_{0}} \sqrt{y_{2}^{2}+z_{2}^{2}} \sin \delta_{2}=B
\end{array}\right\}
$$

$$
\tau_{1}=\frac{\sigma_{1}}{\sigma_{0}} t-\frac{x_{1}}{\sigma_{0}} x_{1}+A \quad \tau_{2}=\frac{\sigma_{2}}{\sigma_{0}} t-\frac{x_{2}}{\sigma_{0}} r_{2}+B
$$

Für gegebene Werte von $y$ und $z$ sind $A$ und $B$ Konstanten, die, wegen der Unabhängigkeit der $y$ und $z$ von der Zeit, auch von dieser unabhängig sind. Beachtet man dies, so zeigt eine einfache Rechnung, daß unsere Transformationsgleichnngen die Gestalt annehmen

$$
\begin{aligned}
& x_{2}=\gamma_{1}^{\prime}\left[x_{1}-u_{12}\left(\tau_{1}-A\right)\right] ; \quad \tau_{2}=\gamma_{1}^{\prime}\left(\tau_{1}+\chi^{\prime} x_{1}+C\right) ; y_{2}=y_{1} ; z_{2}=z_{1}, \\
& \gamma_{1}^{\prime}=\frac{1}{1+\frac{\chi_{1}}{\sigma_{0}} u_{12}}=\frac{1}{\vartheta_{1}^{\prime}} ; \quad C=\frac{\sigma_{2}}{\sigma_{0}} B+A ; \quad \chi^{\prime}=\frac{\sigma_{2} \vartheta_{1}^{\prime 2}-\sigma_{1}}{\sigma_{1} u_{12}} .
\end{aligned}
$$

Beobachten wir also rom System $S_{2}$ aus physikalische Vorgäuge, die sich im System $S_{1}$ abspielen, so findet bezüglich der Transformationsgleichungen eine Anisotropie des Raumes statt, weil $\gamma_{i}^{\prime}$ jetzt von der Richtung abhängt. Diese Anisotropie ist aber, wie wir sehen werden, sehr klein.

Es sei nun das System $S_{2}$ in absoluter Ruhe. Dann haben wir in unseren Formeln (43)

zu setzen, und erbalten

$$
\begin{gathered}
v_{2}=k_{2}=0 ; \quad \sigma_{2}=\sigma_{0} ; \quad \tau_{2}=t ; \\
u_{12}=-u_{21}=-v_{21}=-v_{1}=-v
\end{gathered}
$$

$$
\begin{aligned}
& x_{2}=\frac{x_{1}+v \tau_{1}}{1-\frac{k_{1}}{\sigma_{0}} v} ; \quad y_{2}=y_{1} \\
& \tau_{2}=\frac{\tau_{1}+\frac{k_{1}}{\sigma_{0}} x_{1}}{1-\frac{k_{1}}{\sigma_{0}} v} ; \quad z_{2}=z_{1}
\end{aligned}
$$


Dies ist bis auf Größen dritter Ordnung mit der Lorentztransformation identisch, falls wir setzen $k_{1}=\frac{v_{1}}{\sigma_{0}}$, denn wir erbalten dann:

$$
\left.\begin{array}{ll}
x_{2}=\frac{x_{1}+v \tau_{1}}{1-\frac{v^{2}}{\sigma_{0}^{2}}} & y_{2}=y_{1} \\
\tau_{2}=\frac{\tau_{1}+\frac{v}{\sigma_{0}^{2}} x_{1}}{1-\frac{v^{2}}{\sigma_{0}^{2}}} ; & z_{2}=z_{1}
\end{array}\right\}
$$

In unseren Betrachtungen hielten wir dabei an den durch (27) bis (30) ausgedrückten Symmetriebedingungen fest.

Unter diesen Annahmen gibt also die Transformation (46) an, wie in einem bewegten System sich abspielende physikalische Vorgänge, von einem ruhenden System aus betrachtet, erscheinen, falls in diesem bewegten System die scheinbare Zeit gebraucht wird. Unter der Annahme des Paschskyschen Prinzips ist aber, beim hentigen Stande der experimentellen Wissenschaft, diese Zeit die einzige experimentell faßbare, die wir daher fortwährend gebrauchen. Daher ist auch der Übergang von einem bewegten zu einem ruhenden System nicht durch eine Galileitransformation, sondern durch die Transformation (46) dargestellt. Dabei ist die Lorentztransformation als eine Annäherung anzusehen.

Alles dies gilt aber unter Beibehaltang des absoluten Raumes und der absoluten Zeit.

Wir können mit Hilfe unserer Transformationsgleichungen in üblicher Weise die Frage z. B. nach der longitudinalen oder transversalen Masse eines bewegten Körpers stellen. Für die longitudinale Beschlennigung erhalten wir

$$
\frac{d^{2} x_{2}}{d \tau_{2}^{2}}=\left(\frac{\sigma_{1}}{\sigma_{2}}\right)^{2} \gamma_{1}^{3} \frac{d^{2} x_{1}^{2}}{d \tau_{1}^{2}}
$$

Daraus könnte man analog der Relativitätstheorie auf die Veränderlichkeit der Masse im Verbältnis $\left(\sigma_{1} / \sigma_{2}\right)^{2} \gamma_{1}^{3}$ schließen.

Hier muß aber prinzipiell folgendes zu dieser wichtigen Frage bemerkt werden.

Wir beobachten vom System $S_{2}$ ans zwei auf der $x$-Achse liegende, sich im System $S_{1}$ befindende Körper, und es erbalte jeder dieser Körper unter der Einwirkung des anderen die Beschleunigung $d^{2} x_{1} / d \tau_{1}^{2}$. Vom System $S_{2}$ aus betrachtet wird diese Beschleunigung durch (48) gegeben. 
Es sind nun zwei Annahmen möglich, entweder sind die Massen der Körper im Verbältnis $\left(\sigma_{1} / \sigma_{2}\right)^{2} \gamma_{1}^{3}$ größer geworden, oder die zwischen ibnen wirkende Kraft in demselben Verhältnis kleiner.

Bei dieser Betrachtung gehört, wenn man sich so ausdrücken darf, die auf den Körper wirkende Kraft dem bewegten Systeme an.

Ganz anders aber steht die Frage, weun wir die Beschleunigung eines zu uns relativ bewegten Körpers betrachten, die dieser Körper durch die von ruhenden Körpern ausgeübte Kraft erhält. In diesem Fall kann es geschehen und das ist sogar die allgemeinste Annahme, daß die Kraftwirkung zwischen zwei bewegten Körpern von ihrer gegenseitigen Geschwindigkeit abbängt, und wenn wir die Art dieser Abhängigkeit nicht wissen, können wir nichts über die Veränderlichkeit der Masse aussagen. In dem Fall der Relativitätstheorie steht die Sache etwas anders. Betrachten wir z. B. die Argumentation, welche Herr Einstein in seiner grundlegenden Arbeit ${ }^{1}$ ) anführt, so sehen wir, daß neben der Veränderung der Beschleunigung durch die Lorentztransformation noch die durch die Invarianz der Maxwellschen Gleichungen gegenüber dieser Transformation, bedingte Tatsache vorkommt, daß die von $S$ gedachte ponderomotorische Kraft,

$$
X, \quad Y-\frac{v}{\nabla} N, \quad Z-\frac{v}{\nabla} M,
$$

yon $S^{\prime}$ betrachtet, sich als elektrostatische Kraft $X^{\prime}, Y^{\prime}, Z^{\prime}$, äuBert ${ }^{2}$ ). Dies ist aber nur dann der Fall, wenn wir an der absoluten Richtigkeit der Maxwell-Lorentzschen Gleichungen festhalten. Tun wir das nicht, so können alle Versuche über die elektromagnetische Masse in ganz anderer Weise interpretiert werden, indem man die Abhängigkeit der ponderomotorischen Kraft von der Geschwindigkeit nicht linear annimmt ${ }^{3}$ ). Nehmen wir aber sogar an, daB in unserm Fall die Kraft $X$ sich so transformiert, daß $X_{1}$ gleich $X_{2}$ ist, so daß die Gleichungen

$$
m \frac{d^{2} x_{1}}{d \tau_{1}^{2}}=X_{1} ; \quad m\left(\frac{\sigma_{1}}{\sigma_{2}}\right)^{2} \gamma_{1}^{3} \frac{d^{2} x_{2}}{d \tau_{2}^{2}}=X_{2}=X_{1}
$$

offenbar im Sinne der Veränderung der Masse interpretiert sein müssen. Da die Geschwindigkeit der Erde nur etwa ${ }^{1} 1000$ der Lichtgeschwindigkeit beträgt, so können wir diese Geschwindigkeit in allen Fällen, wo wir mit sehr großen Geschwindigkeiten za tun haben, aunähernd gleich Nall setzen. (Versuche von Hern Bucherer, Hupka u.a.)

\footnotetext{
1) Ann. d. Phys. 17, 891, 1905.

2) 1. c., S. 919 .

3) N. v. Raschewsky, Phys. Zs. 23, 2, 1922. Auch Malmström, Phys.
} ZS. 19, 43, 1918. 
Dann gelten mit großer Annäherung bis in die vierte Deximalstelle die Transformationsgleichungen (46). Durch ihre Anwendung erhalten wir für die longitudinale bzw. transversale Masse Ausdrücke, welche die Funktion $k$ enthalten. Durch die Wahl einer bestimmten Gestalt für $k$ können dann diese Ausdrücke immer mit dem Experiment in Einklang gesetzt werden. In dieser Weise können die Versuche mit sehr schnellen Elektronen zur Aufklärung der Gestalt von $k$ beitragen.

Allerdings können die bisherigen Versuche keineswegs weder als experimentelle Bestätigung der Relativitätstheorie noch als Widerlegung unserer Anschauungen ${ }^{1)}$ angesehen werden.

3. Wir möchten nun noch einiges über den Zusammenhang dieser Betrachtungen mit der allgemeinen Relativitätstheorie und der Ein steinschen Gravitationstheorie vorbringen. Die letzte fußt eigentlich auf drei Grundannahmen:

Annahme I: Eine jede beliebige Bewegung des Körpers verkürzt seine Dimensionen in der Richtung der momentanen Geschwindigkeit, und zwar in dem von der speziellen Relativitätstheorie geforderten Verhältnis; dies gilt auch bezüglich des zeitlichen Verlanfes, der sich in dem bewegten Körper abspielenden Vorgänge.

Annahme II: Ein Gravitationsfeld ist einer Beschleunigung äquivalent.

Annahme III: Die Gravitationsfeldgleichungen müssen invariant gegen beliebige Koordinatentransformationen sein.

Die erste Annahme führt zu dem Schluß, daß eine geodätische Durchmessung des Raumes eines beschleunigten Systems mit beschleunigten Maßstäben eine Abweichung von dem euklidischen Raume geben ${ }^{2}$ ) werde, und zwar wegen der Beeinflussung der Maßstäbe durch die Bewegung. Die Beschleunigung bewirkt also eine Krümmung, eine Verzerrung des Raumes und sogar allgemeiner, der ganzen vierdimensionalen Welt.

Die Annahme II führt dazu, daß eine gleichartige Krümmung im Gravitationsfelde vorhanden ist.

Einstein nimmt an, daß das Gravitationsfeld durch die Komponenten $g_{i k}$ des metrischen Fundamentaltensors vollständig definiert wird, und da die einzige Differentialinvariante zweiter Ordnung der $g_{i k}$ die Riemannsche Krümmung ${ }^{3}$ ) ist, so gelangt man schließlich,

1) Vgl. Prinzipielles dazu von H. Dingler, Phys. ZS. 21, 672-673 und besonders $674,1920$.

2) Vgl. das Einstein sche Beispiel der rotierenden Scheibe.

3) H. Wey ], Raum, Zeit, Materie. Berlin, Bpringer, 1921. Anhang II. 
wenn man noch die Forderung aufstellt, daß die gesuchten Gleichungen zweiter Ordnung ${ }^{1}$ ) seien, nach einigen Überlegungen zu der Einsteinschen Grundgleichung

$$
[\$]_{i}^{k}=-\mathfrak{I}_{i}^{k}
$$

welche die Krümmung der Welt durch die Verteilung des EnergieImpuls-Tensors ausdrückt.

Sind nun die Bahnen der materiellen Körper geodätische Linien, sozusagen nicht-euklidische Gerade in der gekrümmten Welt, so ergeben sich daraus die Gesetze der Planetenbewegungen usw.

Von unserem Standpunkte aus kann ron einer wahren, physischen Kontraktion der Körper durch Bewegung keine Rede sein. In einem beschleunigten System bleiben also unsere Maßstäbe starr ${ }^{2}$ ), und alle geodätischen Abmessungen werden, ganz wie in rubenden System keive Abweichung von dem euklidischen Raume ergeben. Die Beschleunigung verzehrt den Raum nicht. Behält man also die Annahme II, das berühmte Xquivalenzprinzip, bei, so gelangt man nicht mehr zu den Einsteinschen Gravitationsgleichungen.

Daher muß das Åquivalenzprinzip aufgregeben werden, und wir beabsichtigen zu zeigen, daß unter der Voraussetzung allein der Annahme III des Prinzips der allgemeinen Invarianz, man ebensogut zu den Einsteinschen Gleichungen gelangen kann.

Unter der Einwirkung der Gravitation bewegen sich alle Körper auf krummlinigen Bahnen. Darch die Feldgleichungen der Gravitation, kombiniert mit den Bewegungsgleichungen der Körper, sind die Gestalten dieser Bahnen gegeben.

Nun kann aber jede krmmme Linie als geodätische Linie, als nicht-euklidische Gerade, eives entsprechend gekrümmten Raumes angesehen werden; dasselbe gilt natürlich auch für die vierdimensionale Welt.

Führen wir also in unsere Betrachtang als mathematisches Ililfsmittel solch eine fiktiv gekrümmte Welt ein und suchen nach dem Gesetz, welches die Krümmung dieses fiktiven Raumes mit der Verteilung der Materie verknuipft, so gelangen wir, die Annahme III berücksichtigend, nach analogen Überlegungen, wie Einstein, formell zu denselben Gleichungen; die physikalische Interpretation dieser Gleichungen ist aber eine ganz andere. Die $g_{i k}$ siud hier un als das Feld bestimmende Potentiale anzusehen und stehen in keinem Zusammenhang mit der Raummetrik.

1) 1. c., S. 208 .

2) Über den Begriff „starr", vgl. Dingler, Phys. ZS. 21, 487, 1920. Zeitschrift futr Physik. Bd. $X$. 
226 N. v. Raschevsky, Zur Frage nach der physikalischen Bedeutung usw.

Nach dieser Auffassung muß also eine geodätische Ausmessung des Raumes im Gravitationsfelde keine Abweichung vom euklidischen Raume ergeben. Solch eine Abweichung ist auch nicht ausgeschlossen. Würde aber eine genügend genane Messung einmal ergeben, daß z. B. das Verhältnis der Kreislänge zum Durchmesser kleiner als $\pi$ ist, so würden wir den Schluß ziehen ${ }^{1}$ ), daß die. Maßstäbe durch das Gravitationsfeld so beeinflußt werden, daß ein senkrecht zu den Kraftlinien stehender Maßstab länger ist, als derselbe Maßstab parallel zu ihm gelegt. Dieser Einfluß folgt aber keineswegs ans den Feldgleichungen and ist von ihnen ganz unabbängig

Es ist natürlich, wie schon Poincaré2) gezeigt hat, ganz gleichgültig, ob man die enklidische Geometrie beibehält und die physikalischen Gesetze verändert, oder das entgegengesetzte tut.

Wir bemerken noch, daß wegen der Erhaltung der Einsteinschen Feldgleichungen, die auf der Erdoberfläche herrschende Zentrifugalkraft, gleich gut durch die absolute Rotation der Erde, wie durch die relative Bewegung der Fixsternenwelt erklärt werden kann ${ }^{3}$ ).

Doch ist hier wegen der Aufgabe des Äquivalenzprinzips eine experimentelle Entscheidung prinzipiell immer möglich. Allerdings setzt diese Möglichkeit eine andere, nämlich die der unidirektionalen Lichtgeschwindigkeitsmessung voraus. Solange uns diese Möglichkeit entzogen ist, operieren wir nur mit scheinbarer Zeit und scheinbaren Geschwindigkeiten, für welche das klassische Additionstheorem nicht mehr gilt. Daher wird auch die Bewegung eines starren Körpers nicht durch eine Galileische, sondern durch eine Lorentzsche im verallgemeinerten Sinne - Transformation ausgedrückt.

Zum Schluß möchte ich dem Institutsdirektor Herrn Professor Dr. F. Záviška meinen tiefsten Dank aussprechen. Nur durch die liebenswürdige Aufnahme in seinem Institut ist es mir möglich geworden, diese Arbeit auszuführen.

Prag, Institut f. Theoret. Physik d. Böhm. Universität, April 1922.

1) H. Dingler, 1. c., S. 490.

2) H. Poincaré. La science et l'hypothèse. Paris, E. Flammarion, 1918, S. 93 .

3) H. Thirring, Phys. ZS. 19, 33, 1918 ; auch A. Kopff, Phys. ZS. 22, 24 und 179, 1921; vgl. die Kritik dazu von E. Reichenbacher, Phys. ZS. 22, 234, 1921. 